\title{
MEDO E ANSIEDADE DURANTE O TRATAMENTO ODONTOLÓGICO: COMO A PSICOLOGIA PODE AJUDAR?
}

\section{FEAR AND ANXIETY DURING DENTAL TREATMENT: HOW CAN PSYCHOLOGY HELP?}

\author{
Elaine Aparecida Ferreira Machado'; Rodrigo Moreira Caetano Pinto²
}

1 - Discente do Curso de Psicologia na Universidade Unicesumar - Campus Ponta Grossa - PR.

2 - Docente do Centro de Ciências Biológicas e da Saúde da Universidade Unicesumar - Campus Ponta Grossa - PR, Mestrado em Ciências Farmacêuticas pela Universidade Estadual de Ponta Grossa - PR.

\section{RESUMO:}

A ansiedade é caracterizada por uma preocupação excessiva de que algo ruim vai acontecer, associada ao medo, como resposta emocional por uma ameaça real ou imaginária. Muitas pessoas enfrentam ansiedade e medo ao se depararem com os procedimentos odontológicos. O presente estudo dedicou-se a compreender as raízes do sofrimento psicológico durante o atendimento odontológico. Além de identificar os gatilhos que desencadeiam ansiedade, em relação aos procedimentos odontológicos, buscou-se compreender o grau de ansiedade dos pacientes. Estudaram-se, ainda, as possíveis técnicas da terapia cognitivo-comportamental, a serem utilizadas para redução da ansiedade. Quanto a metodologia, classificou-se pela pesquisa de campo e realizou-se por meio de questionários online, após apreciação pelo comitê de ética em pesquisa. A população amostral constituiu-se como parte entre homens e mulheres com a faixa etária entre 16 e 60 anos, que passaram por atendimento odontológico. Outra parte amostral constituiu-se por profissionais de odontologia que fazem atendimento em diferentes especialidades: implantodontia; ortodontia; periodontia; endodontia, cirurgia bucomaxilofacial, cirurgião dentista clínico geral. Constatou-se que o medo e a ansiedade estão presentes nos diversos fatores que antecedem o procedimento odontológico, bem como o profissional dentista deve estar atento as reações do paciente proporcionando um atendimento diferenciado ao mesmo. Demonstrou-se que a psicologia e a odontologia podem criar interfaces em prol da redução da ansiedade dos pacientes.

PALAVRAS-CHAVE: Ansiedade; Psicologia; Medo dentista.

\section{ABSTRACT:}

Anxiety is an emotion characterized by an excessive concern that something bad is going to happen, fear is the emotional response to a real or imagined threat. Many people face anxiety and fear when faced with dental procedures, the present study established itself in understanding the roots of psychological suffering, in dental care.In addition to identifying the triggers that trigger anxiety, in relation to dental procedures, investigating the degree of anxiety of patients, as well as demonstrating the possible techniques of cognitive-behavioral therapy, to be used to reduce anxiety. As for the methodology, it was classified by field research and was carried out through online questionnaires, which was submitted to the research ethics committee. The sample population was formed as part of men and women aged between 16 and 60 years old, who underwent dental care. Another part of the sample consisted of dental professionals who provide care in different specialties: implantology; orthodontics; periodontics; endodontics, maxillofacial surgery, general dentist. It was found that fear and anxiety are present in the various factors that precede the dental procedure, 
as well as the dentist must be attentive to the patient's reactions providing a differentiated service to him. It was identified that psychology together with dentistry can create interfaces in order to reduce patients' anxiety.

KEYWORDS: Anxiety; Psychology; fear dentist.

\section{INTRODUÇÃO}

O tratamento odontológico ainda é visto como um procedimento aversivo para muitas pessoas, devido a experiências de dor ou algum episódio que desencadeou o medo. Este é causado por estímulos que avisam que algo ruim vai acontecer, portanto o medo é aprendido e está associada a dor. Devido a práticas antiquadas da odontologia e acolhimento rudimentar no passado, o consultório do dentista pode ser sinal de sofrimento às pessoas (COELHO et al., 2016).

O medo é uma sensação que está relacionada ao processo emocional que abrange a amígdala e o hipotálamo. Para Fuentes "A amigdala está envolvida no processamento das informações sobre o risco potencial de um evento e pode produzir respostas que levarão ao medo, à fuga ou a paralisação" (FUENTES et al., 2014, p. 44). Já o hipotálamo está ligado as áreas límbicas, ao qual recebe informações do meio interno, podendo atuar de modo direto (FUENTES 2014). Nas literaturas é comum ver a associação entre medo e ansiedade, porém são distintos.

A ansiedade é uma reação diante de uma situação desconhecida, uma resposta emocional que gera preocupação com algo incerto. Quando o sentimento de ansiedade ocorre frente a tratamentos odontológicos, recebe o nome de ansiedade odontológica. Apesar do avanço tecnológico, a ansiedade aparece frente ao barulho da caneta de autorrotação, anestesia ou ainda do barulho do motor de implante nas cirurgias invasivas de implante dentário. A ansiedade odontológica desencadeia respostas no sistema fisiológico e psicológico do paciente, o qual ocorre mudanças no sistema respiratório, aumento da pressão arterial, aumento da frequência cardíaca, aumento da mobilidade intestinal entre outros sintomas (BARASUOL et al., 2016).

cirurgião dentista, ao se deparar com a ansiedade e medo do paciente, procura fazer o controle por meio de recursos e métodos diferenciados que possam reduzir os estímulos que acionam a ansiedade, a fim de melhorar a saúde bucal dos pacientes (COSTA et al., 2013). Dentre os recursos utilizados pelos cirurgiões dentistas estão os sedativos orais, anestésicos, descontração por meio de uma conversa, música, relaxamento facial, até a psicologia, reduzindo assim o estresse e o desgaste do cirurgião dentista frente ao paciente. 
Ainda, o dentista sofre ao se deparar com o paciente ansioso, o que muitas vezes leva à fadiga profissional. Nesse contexto, o cirurgião dentista precisa estar atento ao seu comportamento e suas reações (POSSOBON et al., 2007).

A psicologia, por meio da Terapia Cognitivo-Comportamental (TCC), pode contribuir com o paciente e o profissional cirurgião dentista, ajudando ambos a gerenciarem seus pensamentos, emoções e modo de reagir ao meio. O trabalho da TCC é feito em conjunto com o paciente, identificando, investigando e corrigindo distorções do pensamento que causam sofrimento emocional ao sujeito (KNAPP et al., p.20, 2004).

As intervenções cognitivo-comportamentais utilizadas referem-se a psicoeducação e verificação de pensamentos automáticos/emoções. A psicoeducação é uma ferramenta importante para informar o paciente no seu processo de mudança. Os pensamentos automáticos surgem em paralelo a pensamentos manifestos, muitas vezes vem de forma distorcida quando a ansiedade está presente, por isso é fundamental a verificação desses pensamentos automáticos e emoções e avalia-los posteriormente. (OLIVEIRA, 2011).

Com base no contexto acima, é possível a psicologia atuar em parceria com a odontologia, desenvolvendo intervenções que sejam próprias para cada paciente, levando em conta o comportamento, personalidade e sua subjetividade. A presente pesquisa busca investigar: como a psicologia pode contribuir na redução da ansiedade em pacientes adultos, frente aos procedimentos odontológicos?

A pesquisa tem como objetivo compreender as raízes de sofrimento psicológicos em atendimentos odontológicos, empreendendo técnicas para reduzir a ansiedade frente a esses procedimentos. Identificar os gatilhos que desencadeiam ansiedade, em relação aos procedimentos odontológicos. Investigar o grau de ansiedade dos pacientes e demonstrar as possíveis técnicas da terapia cognitiva-comportamental, a serem utilizadas para redução da ansiedade.

\section{METODOLOGIA}

A pesquisa delimitou-se pelo estudo de campo, que procura aprofundar uma realidade específica. A realização do estudo ocorreu-se por meio da observação direta das atividades do grupo estudado, de entrevistas para compreender as informações e interpretações que ocorrem naquela realidade (GIL, 2002). Como envolve seres humanos, a pesquisa submeteu-se ao comitê de ética em pesquisa, sendo aprovado por parecer consubstanciado nำ4.368.552. 
Constituíram-se como parte amostral homens e mulheres entre 16 e 60 anos de idade que já passaram por atendimento odontológico. Foram excluídos da amostra crianças, adolescentes abaixo de 16 anos e idosos. Outra parte da população amostral constituiu-se por profissionais de odontologia que fazem atendimento em diferentes especialidades, dentre elas implantodontia; ortodontia; periodontia; endodontia, cirurgia bucomaxilofacial, cirurgião dentista clínico geral.

Para obtenção dos dados aplicou-se questionários online através do Google Formulários. O questionário para o paciente teve como objetivo avaliar a intensidade dos sintomas de medo e ansiedade através de autorrelato, e identificar os principais fatores que levam à ansiedade e sofrimento psicológico no atendimento odontológico. O questionário para o profissional dentista teve por objetivo entender a percepção do profissional acerca do sofrimento psicológico do paciente, e identificar ferramentas já aplicadas em seus consultórios a fim de minimizá-lo.

A partir dos dados encont A pesquisa delimitou-se pelo estudo de campo, que procura aprofundar uma realidade específica. A realização do estudo ocorreu-se por meio da observação direta das atividades do grupo estudado, de entrevistas para compreender as informações e interpretações que ocorrem naquela realidade (GIL, 2002). Como envolve seres humanos, a pesquisa submeteu-se ao comitê de ética em pesquisa, sendo aprovado por parecer consubstanciado $\mathrm{n}^{0}$ 4.368.552.

Constituíram-se como parte amostral homens e mulheres entre 16 e 60 anos de idade que já passaram por atendimento odontológico. Foram excluídos da amostra crianças, adolescentes abaixo de 16 anos e idosos. Outra parte da população amostral constituiu-se por profissionais de odontologia que fazem atendimento em diferentes especialidades, dentre elas implantodontia; ortodontia; periodontia; endodontia, cirurgia bucomaxilofacial, cirurgião dentista clínico geral.

Para obtenção dos dados aplicou-se questionários online através do Google Formulários. O questionário para o paciente teve como objetivo avaliar a intensidade dos sintomas de medo e ansiedade através de autorrelato, e identificar os principais fatores que levam à ansiedade e sofrimento psicológico no atendimento odontológico.

O questionário para o profissional dentista, teve por objetivo entender a percepção do profissional acerca do sofrimento psicológico do paciente, e identificar ferramentas já aplicadas em seus consultórios a fim de minimizá-lo.

A partir dos dados encontrados, a discussão da pesquisa versou-se sobre como ferramentas da psicologia cognitiva comportamental podem vir a auxiliar na conduta de 
profissionais da odontologia para o manejo da ansiedade e do medo de seus pacientes, bem como identificar casos em que o tratamento psicológico prévio pode ser uma necessidade. Esses resultados virão a criar interfaces de cooperação entre essas duas áreas, Odontologia e Psicologia, visando proporcionar bem-estar aos pacientes que precisam passar por tratamentos odontológicos.rados, a discussão da pesquisa versou-se sobre como ferramentas da psicologia cognitiva comportamental podem vir a auxiliar na conduta de profissionais da odontologia para o manejo da ansiedade e do medo de seus pacientes, bem como identificar casos em que o tratamento psicológico prévio pode ser uma necessidade. Esses resultados virão a criar interfaces de cooperação entre essas duas áreas, Odontologia e Psicologia, visando proporcionar bem-estar aos pacientes que precisam passar por tratamentos odontológicos.

\section{RESULTADOS E DISCUSSÕES}

\subsection{Abordagens aos pacientes}

Foram entrevistadas 85 pessoas no período de novembro de 2020 à 31 de janeiro de 2021, sendo 67 mulheres e 18 homens. Todos aceitaram por livre e espontânea vontade, participar da pesquisa assinando digitalmente o termo de

consentimento livre e esclarecido. Quanto ao grau de instrução 4 pessoas com ensino fundamental completo, $43 \mathrm{com}$ ensino médio, $10 \mathrm{com}$ ensino superior completo e $28 \mathrm{com}$ pós-graduação.

Sobre o medo de ir ao dentista, $20(23,8 \%)$ pessoas afirmaram terem medo, enquanto $64(76,2 \%)$ afirmaram não ter. Observou-se que o maior público refere-se a mulheres na faixa etária entre 20 anos a 52 anos, evidenciando-se que a idade não é fator predominante. Segundo Pereira et al. (2013), o medo e a ansiedade provocam respostas orgânicas semelhantes, porém a ansiedade não tem causa bem definida como o medo, e este representa uma ameaça real para o indivíduo a nível físico e ao psicológico.

Em relação à desistência/adiamento de um tratamento odontológico por medo ou ansiedade, 19 (22,4\%) pessoas afirmaram que já adiaram ou desistiram do tratamento, 66 $(77,6 \%)$ nunca o fizeram. De acordo com Goes et al. (2010), a ansiedade associada ao tratamento odontológico estimula o estresse entre paciente e dentista, podendo promover o adiamento do tratamento. 
No tocante ao diagnóstico de transtorno Foram entrevistadas 85 pessoas no período de novembro de 2020 à 31 de janeiro de 2021, sendo 67 mulheres e 18 homens. Todos aceitaram por livre e espontânea vontade, participar da pesquisa assinando digitalmente o termo de

consentimento livre e esclarecido. Quanto ao grau de instrução 4 pessoas com ensino fundamental completo, 43 com ensino médio, 10 com ensino superior completo e 28 com pós-graduação.

Sobre o medo de ir ao dentista, 23,8\% das pessoas afirmaram terem medo, enquanto 76,2\% afirmaram não ter. Observou-se que o maior público refere-se a mulheres na faixa etária entre 20 anos a 52 anos, evidenciando-se que a idade não é fator predominante. Segundo Pereira et al. (2013), o medo e a ansiedade provocam respostas orgânicas semelhantes, porém a ansiedade não tem causa bem definida como o medo, e este representa uma ameaça real para o indivíduo a nível físico e ao psicológico.

Em relação à desistência/adiamento de um tratamento odontológico por medo ou ansiedade, 22,4\% das pessoas afirmaram que já adiaram ou desistiram do tratamento e $77,6 \%$ nunca o fizeram. De acordo com Goes et al. (2010), a ansiedade associada ao tratamento odontológico estimula o estresse entre paciente e dentista, podendo promover o adiamento do tratamento.

No tocante ao diagnóstico de transtorno de ansiedade, pânico ou fobia, das pessoas entrevistadas $24,7 \%$ possuíam o diagnóstico, sendo que, 14,1\% afirmaram possuir diagnóstico de outros transtornos psiquiátricos.

Em relação à frequência ao dentista $16,7 \%$ das pessoas frequentam menos que uma vez ao ano, 29,8\% uma vez ao ano, 22,60\% visitam duas vezes ao ano e 9,6\% apenas três vezes ao ano, $21,4 \%$ das pessoas procuram o dentista somente em caso de urgência. Segundo Chaves et al. (2006) no meio de indivíduos mais ansiosos, encontram-se aqueles que não costumam realizar consultas regularmente e quando fazem, são movidos pela dor.

Sobre os fatores que contribuem para a ansiedade relacionada ao atendimento odontológico, os resultados encontram-se na Tabela 1.

Identificou-se que os fatores que contribuem para a ansiedade $96,4 \%$ das pessoas sentem medo de dor nos procedimentos odontológicos, 59\% sofrem com o barulho dos equipamentos, 57,8\% relacionam-se ao impacto mecânico na boca por meio das extrações, implantes, brocas, gerando ansiedade e medo. A recuperação pós-procedimento é fator gerador de ansiedade sendo $24,1 \%$ das pessoas sentem medo de sangramento no póscirurgico. Em consonância com Bottan (2007), o ruído da alta rotação produz desconforto ao paciente ansioso, despertando sensibilidade orgânica e tornando a experiência ruim, o 
qual gera o medo.de ansiedade, pânico ou fobia, 21 (24,7\%) pessoas possuíam 12 (14,1\%) afirmaram possuir diagnóstico de outros transtornos psiquiátricos.

Em relação à frequência ao dentista $16,7 \%$ das pessoas frequentam menos que uma vez ao ano, 29,8\% 01 vez ao ano, 22,60 visitam 02 vezes ao ano e 9,6\% apenas 3 vezes ao ano, $18(21,4 \%)$ pessoas procuram o dentista somente em caso de urgência. Segundo Chaves et al. (2006) no meio de indivíduos mais ansiosos, encontram-se aqueles que não costumar realizar consultas regularmente e quando fazem, são movidos pela dor.

Sobre os fatores que contribuem para a ansiedade relacionada ao atendimento odontológico, os resultados encontram-se na Tabela 1.

Tabela 1. Fatores que contribuem para ansiedade relacionada ao atendimento odontológico

Dos fatores abaixo, quais deles você acha que mais contribui para a ansiedade relacionada ao atendimento odontológico?

\begin{tabular}{lcc}
\hline Alternativas & Pessoas entrevistadas & Porcentagem \\
\hline Medo de sentir dor/sangrar & 84 & 96,40 \\
Barulho dos equipamentos & 49 & 59,00 \\
Impacto mecânico na boca & 48 & 57,80 \\
Medo da recuperação pós-procedimento & 20 & 24,10
\end{tabular}

Fonte: Autoria própria (2021)

Em relação aos procedimentos que os pacientes pontuam como os principais adotados pelos dentistas para redução da ansiedade, os resultados estão expressos na Tabela 2.

Tabela 2. Sobre ações dos dentistas para tornar confortável o atendimento odontológico

O que você acha que os consultórios e dentistas poderiam fazer no sentido de tornar o atendimento odontológico mais confortável?

\begin{tabular}{lcc}
\hline Alternativas & Pessoas entrevistadas & Porcentagem \\
\hline Explicação sobre os procedimentos & 27 & 42,90 \\
realizados. & 09 & 14,30 \\
Pontualidade & 14 & 22,40 \\
Humanização e respeito & 04 & 06,50 \\
Redução do barulho dos equipamentos & 11 & 17,60 \\
Ambiente aconchegante & & \\
\hline
\end{tabular}

Fonte: Autoria própria (2021) 
Em relação aos investimentos que os dentistas fazem $70,6 \%$ orientam o paciente sobre os cuidados e métodos que minimizam sangramentos, corroborando com a expectativa dos pacientes, garantindo dessa forma a tranquilidade de uma recuperação pós-procedimento sem causar ansiedade e desconforto. Segundo Bottan (2007) é importante fornecer explicações claras sobre os procedimentos para que o tratamento odontológico seja efetivo.

\subsection{Abordagem aos Cirurgiões Dentistas}

Foram entrevistados 17 profissionais da odontologia com as especializações em Cirurgia bucomaxilofacial e Implantodontia, Cirurgião clínica geral, Endodontia, prótese e Dentística, com experiência profissional entre um a vinte e três anos.

Com relação a acreditar que os pacientes têm medo de ir ao dentista, dos cirurgiões dentistas entrevistados, $88,2 \%$ acreditam que sim, enquanto $11,8 \%$ acreditam que não e $88,2 \%$ vivenciaram desistência dos pacientes por conta do medo.

Em relação a avaliar se os seus pacientes têm diagnóstico de transtorno de ansiedade/pânico/fobia ou outro transtorno psiquiátrico, 88,2\% dos profissionais revelaram fazer essa avaliação; 82,4\% acreditam que a explicação detalhada do procedimento na maior parte das vezes reduz a ansiedade, pois o paciente sente segurança no profissional.

No tocante a ansiedade do paciente impactar na ansiedade do profissional, $38 \%$ dos profissionais responderam não impactar; 44\% relataram que impacta, 44\% procuram conversar com o paciente para tentar que o mesmo mude sua atenção e $13 \%$ fazem terapia e realizam a técnica da respiração. Em consonância com Murrrer e Francisco (2015) o cirurgião dentista precisa ter cuidado com o paciente adulto utilizando técnicas apropriadas de comunicação para aumentar a confiança e sensação de controle dos mesmos.

Sobre o investimento que os profissionais realizam para reduzir ansiedade de seus pacientes, os resultados estão expressos na tabela 3. 
Tabela 3. Itens que reduzem a ansiedade de pacientes

Dos itens abaixo, em quais deles você investe para reduzir a ansiedade de seus pacientes relacionada ao procedimento odontológico?

\begin{tabular}{lcc}
\hline Alternativas & Cirurgiões Dentistas & Porcentagem \\
\hline Sala espera aconchegante & 10 & 58,90 \\
$\begin{array}{l}\text { Conversar com o paciente, explicando } \\
\text { sobre o procedimento }\end{array}$ & 16 & 94,20 \\
$\begin{array}{l}\text { Estabelecer intimidade com o } \\
\text { paciente }\end{array}$ & 14 & 82,40 \\
$\begin{array}{l}\text { Tranquilizar s/ recuperação pós- } \\
\text { procedimento }\end{array}$ & 12 & 70,60 \\
\hline
\end{tabular}

Fonte: Autoria própria (2021)

Verificou-se que a maior parte dos profissionais mantém um contato mais próximo e usam a comunicação efetiva como estratégia, em detrimento de investimentos na infraestrutura do consultório. Observou-se que a sala de espera, falta de atendimento na hora marcada, cheiro dos consultórios, são fatores ansiogênicos aumentando o medo. Cerca de $58,9 \%$ dos profissionais dentistas investem em recepção e em salas aconchegantes com aromas para tranquilizar o paciente. Segundo Ferreira et al. (2004), indivíduos ansiosos apresentam para o dentista dificuldades de cooperação, relacionamento prejudicado com profissional e necessidades de cuidados durante os procedimentos clínicos.

Constatou-se que os pacientes esperam dos dentistas um relacionamento de intimidade, confiança, segurança ao transmitir explicações sobre os procedimentos e que possam receber um tratamento humanizado. Muitos dos profissionais entrevistados procuram ser atenciosos com seus pacientes ouvindo-os e compreendendo-os em seus sofrimentos. De acordo com Possobon et al. (2007), o cirurgião dentista busca recursos técnicos modernos para amenizar a dor do paciente, porém não se preocupa em buscar conhecimentos psicossocial do paciente, assim sua habilidade para controle da dor não acompanha o avanço tecnológico.

Sobre ações a serem feitas pelos profissionais dentistas relacionados ao atendimento odontológico. Os resultados estão apresentados na Tabela 4. 
Tabela 4. Ações dos dentistas para tornar o atendimento odontológico mais confortável

O que você acha que os consultórios e dentistas poderiam fazer no sentido de tornar o atendimento odontológico mais confortável?

\begin{tabular}{lcc}
\hline Alternativas & Cirurgiões dentistas & Porcentagem \\
\hline Explicar s/ procedimento & 05 & 31,30 \\
Sedação & 05 & 31,30 \\
Ambiente confortável & 04 & 26,00 \\
Música, filmes e séries & 05 & 31,30 \\
$\begin{array}{l}\text { Conversar e ouvir com } \\
\text { atenção o paciente. }\end{array}$ & 06 & 37,50 \\
\hline
\end{tabular}

Fonte: Autoria própria (2021)

Nota-se que o cirurgião dentista procura formas para ajudar o paciente a se sentir confortável em um ambiente que seja acolhedor e que proporcione condições de aumentar a autoestima dele. Em concordância com Kanegane et al. (2006), os cirurgiões dentistas precisam encontrar meios para reduzir a exposição de estímulos que provocam ansiedade para transformar o tratamento em experiência positiva, melhorando a saúde bucal do indivíduo.

Observa-se por meio da pesquisa e dos dados coletados que a psicologia cognitivacomportamental pode auxiliar os profissionais dentistas na conduta de seus próprios sentimentos e poder manejar o controle de ansiedade dos pacientes. Segundo Knapp \& colaboradores (2004), a técnica do relaxamento o de grupos musculares para agilizar a percepção de si durante as crises; técnica de respiração pausada com ritmos sequenciais para aumentar a oxigenação do corpo, tem assumido lugar de destaque para tratamentos eficazes.

\section{CONSIDERAÇÕES FINAIS}

Nota-se que o cirurgião dentista procura formas para ajudar o paciente a se sentir confortável em um ambiente que seja acolhedor e que proporcione condições de aumentar a autoestima dele. Em concordância com Kanegane et al. (2006), os cirurgiões dentistas precisam encontrar meios para reduzir a exposição de estímulos que provocam ansiedade 
para transformar o tratamento em experiência positiva, melhorando a saúde bucal do indivíduo.

Observa-se por meio da pesquisa e dos dados coletados que a psicologia cognitivacomportamental pode auxiliar os profissionais dentistas na conduta de seus próprios sentimentos e poder manejar o controle de ansiedade dos pacientes. Segundo Knapp \& colaboradores (2004), a técnica do relaxamento de grupos musculares para agilizar a percepção de si durante as crises; técnica de respiração pausada com ritmos sequenciais para aumentar a oxigenação do corpo, tem assumido lugar de destaque para tratamentos eficazes.

\section{REFERÊNCIAS}

BARASUOL, J.C. et al. Abordagem de Pacientes com Ansiedade ao Tratamento Odontológico no Ambiente Clínico. Revista Associação Paulista Cirurgião Dentista. São Paulo: v.70, n.1, p. 76-81, 2016.

BOTTAN, E. R.; OGLIO, J. D.; ARAUJO, S. M. de. Ansiedade ao tratamento odontológico em estudante do ensino fundamental. Pesq. Bras. Odontoped. Clin. Integr., Joao Pessoa: v.7, n. 3, p. 241-246, set./dez, 2007.

CHAVES, A.M. et al. Estudo epidemiológico da ansiedade dos pacientes ao tratamento odontológico. Rev. odontol. UNESP, Marília: v. 35, n. 4, p. 263-268, 2006.

COELHO, A.N.M. et al. Influência do Medo No Tratamento Odontológico. FACS/Univale. Rio de Janeiro: 2016.

COSTA, A.M.D.D. et al. Ansiedade Pré-Implante Dentário. Rev.odonto, Odontologia Clinico-cientifica, vol 12, n.4, 2013.

FERREIRA, C. M. et al. Ansiedade odontológica: nível, prevalência e comportamento. RBPS, v. 17, n. 2, p. 51-55, 2004.

FUENTES, D. Neuropsicologia, teoria e prática. 2 ed. Porto Alegre: Artmed, 2014. 
GIL, A.C. Como Elaborar Projetos de Pesquisa. 4 ed. São Paulo: Atlas, 2002.

GOES, M. P. S. et al. Ansiedade, medo e sinais vitais dos pacientes infantis. Odonto.clin.cient. Recife: v. 40, p. 39-44, jan./mar, 2010.

KNAPP, P. Terapia Cognitivo-Comportamental na prática psiquiátrica. Porto Alegre: Artmed, 2004.

KANEGANE, K.; PENHA, S.S.; BORSATTI, M.A. ROCHA, R.G. Ansiedade ao tratamento odontológico no atendimento de rotina. RGO, Porto Alegre: v.54, n.2, p.111-4, abr./jun, 2006.

MURRER, R. D.; FRANCISCO, S. S. Diagnóstico e Manejo da Ansiedade Odontológica pelos Cirurgiões-Dentistas. Curitiba: Interação Psicol., v.19, n.1, p.37-46, jan-abr. 2015.

OLIVEIRA, M. I. Intervenção Cognitivo-Comportamental em Transtorno de Ansiedade: Relato de Caso. Revista Brasileira de Terapias Cognitivas, Aracajú: V.7, n.1, p.30-34, 2011. PEREIRA, V. Z. et al. Avaliação dos níveis de ansiedade em pacientes submetidos ao tratamento odontológico. Rev bras ci Saúde, v. 17, n. 1, p. 55-64, 2013.

POSSOBON, R.F. et al. O Tratamento Odontológico como Gerador de Ansiedade. Psicologia em Estudo, Maringá: v.12, n.3, p.609-616, set/dez, 2007.

Autor para correspondência:

Elaine Aparecida Ferreira Machado

Universidade Unicesumar - Campus Ponta Grossa - PR.

Email: efmachado9@gmail.com

Recebido: 04/06/2021 Aceite: 02/09/2021 